\title{
Quantum Chemical Studies of the Myosin ATPase Mechanism
}

\author{
Hiroshi Kagawa \\ Department of Physics, Nippon Medical School
}

\begin{abstract}
Muscle contraction is one of the molecular motions in the living body. The elemental process of muscle contraction is a mutual sliding of two kinds of filaments of proteins, myosin and actin, using energy liberated by ATP hydrolysis. Myosin enzymatically hydrolyzes ATP and interacts with actin. This article reviews previous quantum chemical studies to help determine how the chemical energy of ATP hydrolysis is converted to mechanical energy during sliding.

(J Nippon Med Sch 2007; 74: 4-10)
\end{abstract}

Key words: myosin, ATP hydrolysis, quantum chemistry, molecular orbital, molecular dynamics

\section{Introduction}

Living bodies have biological molecular motors made of proteins. There are linear motors, such as myosin sliding on an actin filament, dynein and kinesin sliding on a microtubule, and RNA polymerase and DNA helicase sliding on DNA; and there are rotary motors, such as $F_{1}$-ATPase and bacterial flagella ${ }^{1-6}$. Most biological molecular motors utilize chemical energy from adenosine triphosphate (ATP) hydrolysis for their various biological motions. Myosin has long been known to play a role in muscle contraction and has more recently been reported to participate in various intercellular motions, such as chemotaxis, cytokinesis, pinocytosis, and vesicle transport ${ }^{1}$.

ATP supplies energy to all activities of living bodies. Living bodies utilize free energy obtained by hydrolyzing ATP for various motions, ion transport, and chemical reactions ${ }^{7}$. Although proteins principally participate in these actions, energy is also necessary for these proteins to function. Most actions directly utilize energy from ATP hydrolysis, as is the case with muscle contraction.

Myosin, one of the principal proteins of muscle contraction, acts as an enzyme in the hydrolysis of ATP and converts the chemical energy from this hydrolysis to mechanical energy for sliding. This is achieved via the interaction with another principal protein, actin. Although muscle contraction has been studied extensively, the molecular mechanism of the sliding motion of the two kinds of filaments of polymerized myosin and polymerized actin is not well understood. Neither do we fully understand how the chemical energy from ATP hydrolysis is converted to mechanical energy for the sliding motion.

Many experimental and theoretical studies have been carried out to elucidate the molecular mechanism of the sliding in muscle contraction, and many reviews of these studies have also been

Correspondence to Hiroshi Kagawa, Department of Physics, Nippon Medical School, 2-297-2 Kosugi-cho, Nakaharaku, Kawasaki, Kanagawa 211-0063, Japan

E-mail: kagawa@nms.ac.jp

Journal Website (http://www.nms.ac.jp/jnms/) 


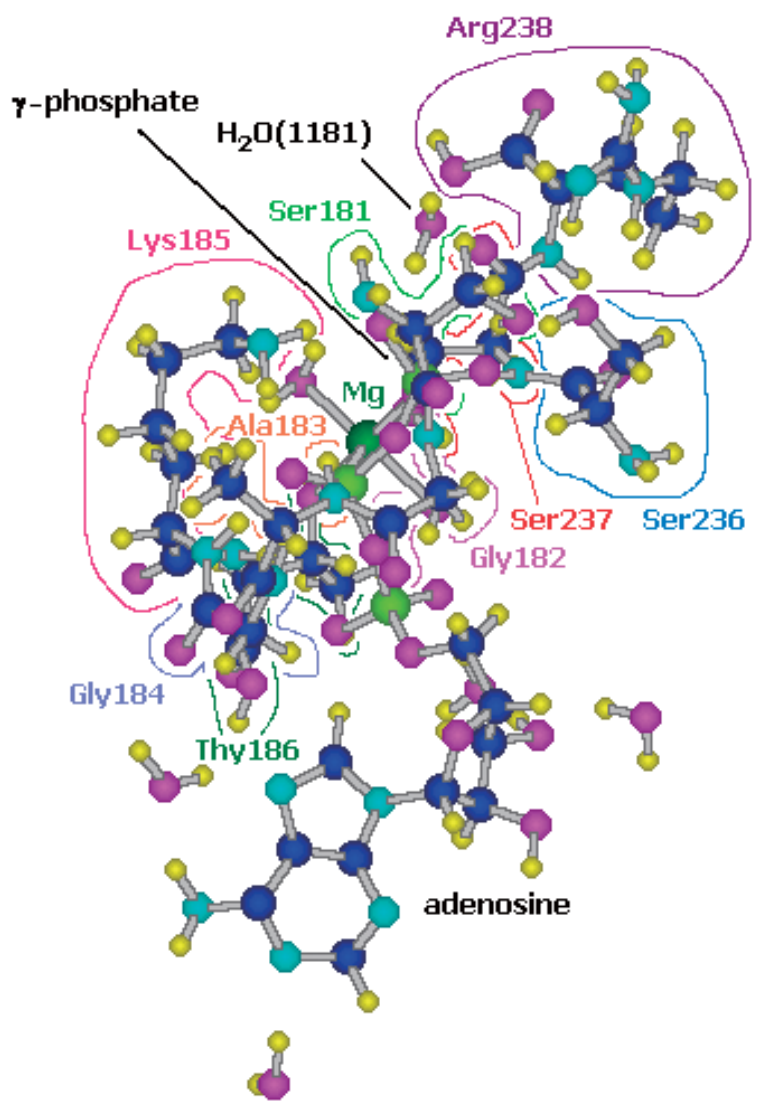

Fig. 1 Molecular structure of the model complex for MgATP-S1Dc determined by positional optimization of hydrogen atoms. All atoms are shown as balls, and bonds and coordinations are shown as sticks. Ball colors denote the species of atom. Dark blue: carbon; blue: nitrogen; red: oxygen; light green: phosphorus; green: magnesium; yellow: hydrogen. The gray sticks signify chemical bonds and coordinations. The number 1181 of the lytic water and the numbers of amino acids correspond to the PDB 1MMD datum.

performed $^{8-10}$. These studies have used various methods, including physiological methods, biochemical methods, and structural methods using optical, electronic, and atomic force microscopes. Other studies have employed methods such as x-ray diffraction, fluorescence energy transfer, and singlemolecule manipulation. The majority of the theoretical studies present hypotheses involving a molecular mechanism to account for the sliding of muscle contraction.

To understand the sliding motion as a molecular mechanism, it is necessary to follow the structural changes in the proteins participating in the motion as well as the specific mechanism of their interaction. It has been well known that the site of ATP binding and hydrolysis and the site of actin binding are different in the myosin molecule ${ }^{8-10}$. For a more complete understanding of the process, the molecular level of energy utilization must be studied in terms of electron and proton transfer. For example, just as the electron transport system is essential in ATP synthesis in mitochondria, it should be similarly approached to understand the utilization of the energy from ATP hydrolysis in terms of electron and proton transfer. A quantum chemistry approach is well suited to this sort of analysis. Since myosin is large, it is difficult to treat the whole molecule quantum chemically, even with the advent of advanced computational technology. Therefore, we have focused on ATP hydrolysis in myosin as a local problem. This review describes our quantum chemical studies of ATP hydrolysis with reference to previous studies by us and by other groups.

\section{Quantum Chemical Studies}

This section briefly reviews previous quantum chemical studies of ATP hydrolysis in myosin, although the relative recency of this approach has allowed few such studies to date. Since it is difficult to study the whole molecule of large protein quantum chemically, simulations by molecular dynamics (MD) using classical mechanics were commonly used. Since, in the case of chemical reactions, a quantum mechanical (QM) treatment is required, this approach has been employed. Furthermore, to simulate the motion of molecules, Monte Carlo methods and MD have been widely used. Monte Carlo methods obtain calculation results obeying statistical stochastic distribution by using random numbers ${ }^{11}$. MD simulates atomic and molecular motion by calculating forces between atoms and molecules with the potential energy of forces between atoms ${ }^{12,13}$.

Molecular orbital studies of ATP and ADP alone or with a metal cation have been performed over many years ${ }^{14-19}$. In a previous study, we used a personal computer and software designed to allow a semiempirical molecular orbital method ${ }^{20,21}$, termed 


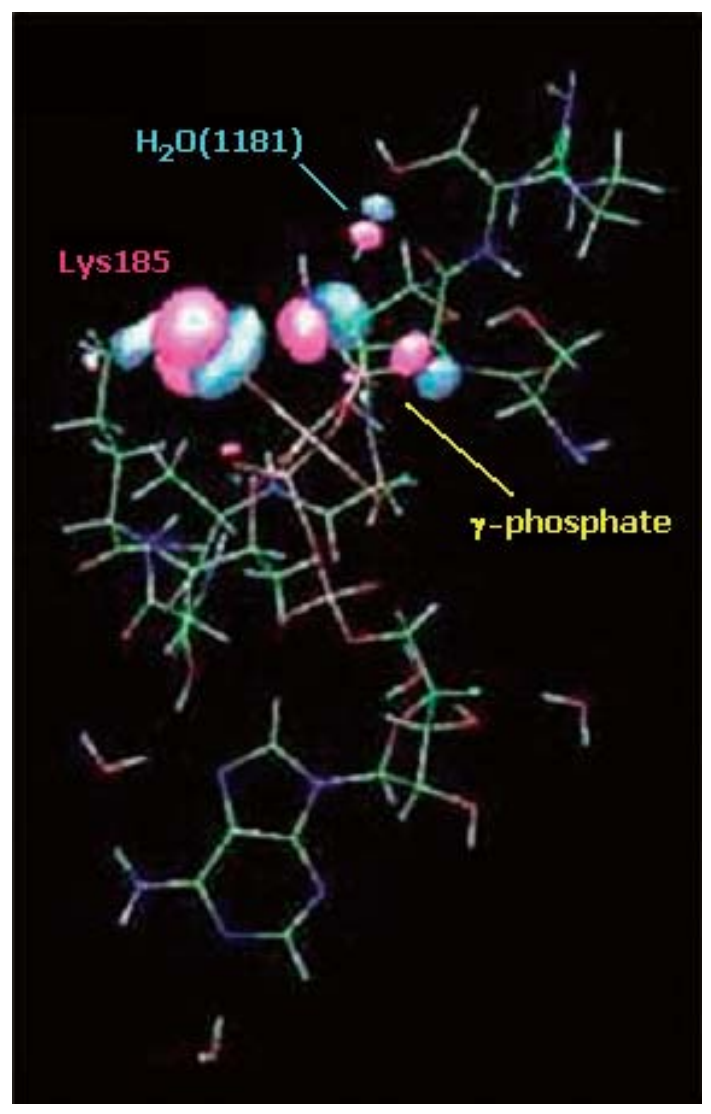

Fig. 2 HOMO of the model complex for MgATPS1Dc. Lobes of HOMO appear on the lytic water $\mathrm{H}_{2} \mathrm{O}$ (1181), two nonbridging oxygen atoms of $\gamma$-phosphate, and the side chain $(\mathrm{N} \zeta$ and $C_{\varepsilon}$ ) of Lys185. Red and blue colors of lobes represent opposite signs of molecular orbital phases. The number 1181 of the lytic water and the number 185 of Lys185 correspond to the PDB $1 \mathrm{MMD}$ datum.

MOPAC $6^{22}$, which, with the obtained local minimum structures of ionized ATP and MgATP depending on the $\mathrm{pH}$ of its environment, enabled us to estimate the acid dissociation constant and difference in free energy due to hydrolysis ${ }^{23}$. Although this approach did not yield experimental values, it became the foundation of our subsequent studies.

\section{A Novel Myosin ATPase Study}

Our novel molecular orbital study ${ }^{24}$ was focused on the initial phase of ATP hydrolysis in myosin using a personal computer, with particular attention to reactive electrons in the frontier molecular orbital theory proposed by Fukui ${ }^{25,26}$. In the analysis of the electronic states of a model structure of MgATP and

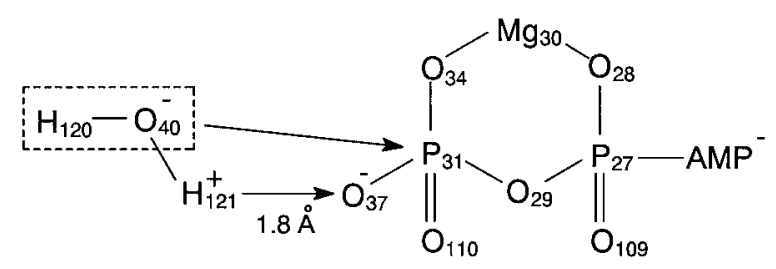

Fig. 3 Schematic diagram of a mechanism of the initial part of ATP hydrolysis. The lytic water $\mathrm{H}_{2} \mathrm{O}$ (1181) $\left(\mathrm{H}^{+}\right.$and $\left.\mathrm{OH}^{-}\right)$binds directly to $\gamma$-phosphate while ATP functions as a base. The proton $\mathrm{H}_{121}^{+}$of $\mathrm{H}_{2} \mathrm{O}$ (1181) transfers to $\mathrm{O}_{37}$, a nonbridging $\gamma$-phosphoryl oxygen atom, and the remaining $\mathrm{OH}^{-}$of $\mathrm{H}_{2} \mathrm{O}$ (1181) transfers to $\gamma$-phosphorus $\mathrm{P}_{31}$. The number 1181 of the lytic water corresponds to the PDB 1MMD datum. The suffix numbers of atoms are serial numbers in the model complex for PM3 calculations.

the surrounding portion of myosin (Fig. 1) using the $\mathrm{x}$-ray crystal structure of the myosin motor domain bound to the stable MgATP analogue, we found that the frontier molecular orbitals indicate the reaction site of ATP hydrolysis, namely, the non-bridging $\gamma$ phosphoryl oxygen atoms, the lytic water $\mathrm{H}_{2} \mathrm{O}$ (1181), and the amino acid Lys185 in the myosin motor domain that is thought to participate in the hydrolysis. The highest occupied molecular orbital (HOMO), one of the frontier molecular orbitals, of the model complex is shown in Figure 2. From this analysis, we proposed hypothetical mechanisms of the initial phase of the hydrolysis, the very initial part of which is presented in Figure 3. This previous experience provided the framework for our quantum chemical approach to the study of muscle contraction.

For this work, we used a semiempirical molecular orbital program, the PM3 method ${ }^{27-29}$ in MOPAC $97^{30}$. The calculations for an $a b$ initio (nonempirical) molecular orbital method are time-consuming but can be applied to a very small system with a high degree of accuracy. In contrast, the semiempirical method can be applied to a larger system using empirical parameters. Fortunately, the rapid increase in computer processing capabilities has allowed us to apply quantum chemical methods to systems as large as biomolecules. However, due to the limited number of atoms available in the former WinMOPAC ${ }^{30}$, in which the MOPAC 97 program 
was implemented, and the low performance of our personal computer, we were able to treat only a small part of the myosin. The original structure of our model was an x-ray crystal structure that is registered as $1 \mathrm{MMD}^{31}$ in the Protein Data Bank (PDB).

In the $\mathrm{PDB}$, there are various $\mathrm{x}$-ray crystal structures of the myosin motor domain, which is a domain having ATP-hydrolysis and actin-binding sites. The x-ray crystal structures ${ }^{31-33}$ of the myosin motor domain of a soil-living amoeba, Dictyostelium discoideum, were obtained using various ATP analogues or ADP as a bound nucleotide. As structures of the prehydrolysis state (ATP bound state), there are $1 \mathrm{MMG}^{32}(\mathrm{MgATP} \gamma \mathrm{S}), 1 \mathrm{MMN}^{32}$ (MgAMPPNP), $1 \mathrm{MMA}^{32}$ (MgADP), and $1 \mathrm{MMD}^{31}$ $\left(\mathrm{MgADP}-\mathrm{BeF}_{x}\right)$; and, for the transition state, there are $1 \mathrm{MND}^{31}\left(\mathrm{MgADP}-\mathrm{AlF}_{4}\right)$ and $1 \mathrm{VOM}^{33}(\mathrm{MgADP}$ $\left.\mathrm{VO}_{4}\right)$.

The original structure of the model used in our work was 1MMD, the three-dimensional structure of MgADP-BeF ${ }_{x}$ S1Dc, which is a complex of the MgATP analogue and the myosin motor domain of D. discoideum myosin II, as determined by Fisher, et $\mathrm{al}^{31}$ using x-ray crystallography. The MgATP analogue, $\mathrm{MgADP}-\mathrm{BeF}_{x}$, is stable, so that $\mathrm{MgADP}$ $\mathrm{BeF}_{x}$-S1Dc was used as an analogue of MgATP-S1Dc for x-ray crystallography. This structure consists of ca. 6,000 atoms, including the ATP analogue and oxygen atoms of water.

For the quantum chemical study, we extracted only $\operatorname{MgADP}-\mathrm{BeF}_{x}(x=3$ in the PDB 1MMD data), 9 amino acid residues, and 7 oxygen atoms of water near the ATP analogue, as shown in Figure 1. To restructure the $\mathrm{MgADP}-\mathrm{BeF}_{3}$ to be a model of MgATP, the beryllium atom, Be, was replaced with a phosphorus atom, $\mathrm{P}$, and the fluorine atoms, $\mathrm{F}_{3}$, were replaced with oxygen atoms, $\mathrm{O}_{3}$. Furthermore, hydrogen atoms missing in the $\mathrm{x}$-ray structures were added to the model structure, $\mathrm{OH}$ was added to each C-terminal of the amino acid chains, and 2 hydrogen atoms were added to each N-terminal. Only hydrogen atoms and the added $\mathrm{OH}$ were optimized to obtain minimal energy structures with the PM3 method in MOPAC 97. This model structure consisted of only 186 atoms.
Calculations were performed on the model structure as a whole and as two separate parts. The optimized structure, called the "model complex," was separated into two parts: MgATP-2w (termed ligand here) and cage (termed receptor here), so that we could analyze interactions between these two parts. The $2 \mathrm{w}$ in MgATP-2w indicates 2 water molecules that coordinate tightly with the $\mathrm{Mg}^{2+}$ ion. Therefore, these were included in the MgATP portion. The receptor (cage) is composed of 9 amino acid residues and the other 5 water molecules.

We calculated with MOPAC and analyzed changes in the total energy, orbital energies, and atomic net charges due to the complex formation. Furthermore, we made and analyzed a molecular orbital correlation diagram (Figure 2 shows a main part of the diagram) and considered the interaction between the ligand and the receptor from an electro-theoretical perspective. Then, the interaction between the ligand and the receptor was seen to induce a charge transfer to the antibonding molecular orbital of the lytic water and then to promote weakening of the $\mathrm{O}-\mathrm{H}$ bond of the lytic water to induce ATP hydrolysis. We concluded that this was the first event in hydrolysis. Additionally, our calculation results indicated that Lys185 plays an important role in the mixture of the electronic states.

\section{Subsequent Studies by Other Groups}

Studies using high-performance computers by other groups ${ }^{34-36}$ followed our initial study. These studies treated the whole myosin motor domain, although it is impossible to quantum chemically calculate the whole domain; thus, they used classical mechanics to make MD simulations for the whole system. They also studied only a small part of the active site, namely, the phosphates of ATP and the side chains of its surrounding amino acids, with calculation methods that employed QM. Since they used time-consuming, $a b$ initio methods, the quantum calculations were limited to very small regions. This combined approach using MD, with QM used for the active region and classical molecular mechanics (MM) for the majority of the remainder to estimate 
the potential between atoms, has been termed the $\mathrm{QM} / \mathrm{MM}-\mathrm{MD}$ method.

Okimoto, et al. ${ }^{34}$ used Gaussian $98^{37.38}$ for quantum calculations and the Amber program (version 5.0, University of California, San Francisco) ${ }^{39}$ for MD calculations. They used 1VOM as the model structure, which is thought to be a transition state, replaced $\mathrm{VO}_{4}$ with $\mathrm{PO}_{3}+\mathrm{H}_{2} \mathrm{O}$, and repaired amino acids that were missing in the PDB datum. They concluded that the ATP hydrolysis in myosin is a single elementary reaction with Walden inversion of $\gamma$-phosphate, and suggested that amino acids play important roles: Lys185 maintains the structure of the $\beta$ - and $\gamma$-phosphates of ATP with hydrogen bonds, Thr186 and Ser237 are coordinated to $\mathrm{Mg}^{2+}$ and stabilize the ATP conformation, and Arg238 and Glu459 retain the lytic water at the appropriate position for the hydrolysis.

Minehardt, et al. ${ }^{35}$ used an in-house version of the Car-Parrinello ${ }^{40}$ code, CP90, for quantum calculations and the Amber program (version 6.0, University of California, San Francisco) $)^{39,41}$ for MD calculations. As in our previous study, they also used 1MMD corresponding to the prehydrolysis state and used $1 \mathrm{BR} 4^{42}$ made of chicken gizzard myosin with ADP$\mathrm{BeF}_{3}$, which corresponds to the transition state, after $\mathrm{BeF}_{3}$ is replaced with $\mathrm{PO}_{3}$. They also considered the roles of amino acids surrounding ATP between the prehydrolysis and transition states, the possible roles of a salt bridge between Arg238 and Glu459 as a gate of ATP, and the roles of products of the ATP catalytic pocket. They calculated both cases of MgATP and CaATP, and suggested that higher CaATPase rates occur because the leaving phosphate group is more weakly bound and dissociation occurs faster. Finally, their conclusions differed from those we made based on our estimations, which they discussed in their report. Specifically, their conclusions differed in terms of the destination of $\mathrm{H}^{+}$of the lytic water and the role of Lys185 in the ATP hydrolysis.

Schwarzl, et al. ${ }^{36}$ used the GAMESS ${ }^{43,44}$ for quantum calculations and $\mathrm{CHARMM}^{45,46}$ for $\mathrm{MD}$ calculations. They used an unpublished structure with $\mathrm{MgADP}-\mathrm{BeF}_{3}$ after $\mathrm{BeF}_{3}$ is replaced with $\mathrm{PO}_{3}$, and discussed three possible initial reaction

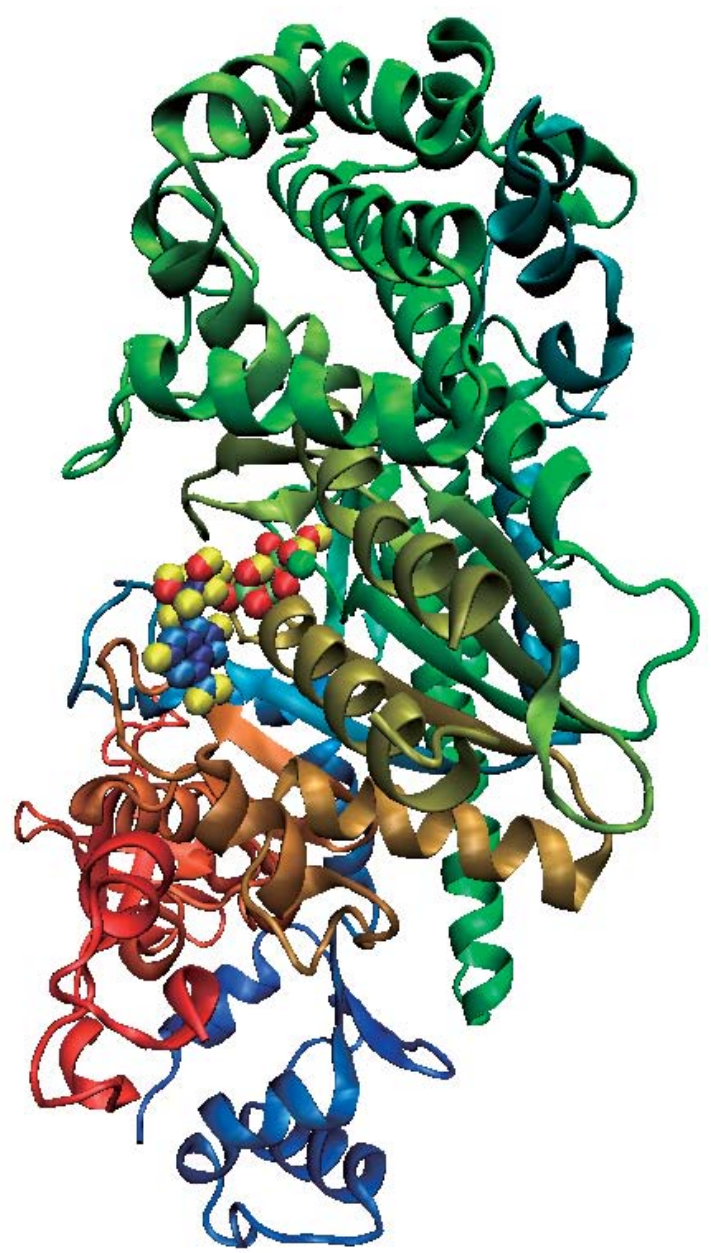

Fig. 4 Ribbon representation of the motor domain of $D$. discoideum myosin II (PDB 1MMD). MgATP and the lytic water (located at the upper end of MgATP) are shown as spacefilling models where the color of spheres denotes the species of atom. Dark blue: carbon; blue: nitrogen; red: oxygen; light green: phosphorus; green: magnesium; yellow: hydrogen. Ribbon colors denote segments not mentioned in this article.

pathways for ATP hydrolysis and processes thereafter. The pathways are similar to those proposed by $\mathrm{us}^{24}$, in which the proton of the lytic water transfers to a nonbridging oxygen of $\gamma$ phosphate directly or via one of the surrounding amino acids of $\gamma$-phosphate. According to their results, the three initial pathways are equally likely.

A study by Onishi, et al. ${ }^{47}$ offers a good framework for understanding our most recent study. They estimated the process of ATP hydrolysis based on point mutation from their own studies ${ }^{48-50}$ and based on the structures of the prehydrolysis and transition 
states as determined by Rayment's group ${ }^{31,33}$. In their findings, the destination of $\mathrm{H}^{+}$of the lytic water differs from that in our study: we estimated that the destination is a nonbridging oxygen of $\gamma$-phosphate, whereas they proposed that it is a water molecule close to the lytic water, which becomes an oxonium ion.

\section{Our Recent Study}

We recently initiated $\mathrm{QM} / \mathrm{MM}-\mathrm{MD}$ simulations similar to those by Okimoto, et al., Minehardt, et al., and Schwarzl, et al. with a high-performance computer, using the whole myosin motor domain with MgATP converted from its analogue, as shown in Figure 4. Because of the time-intensive nature of $a b$ initio methods, we have not used them for QM calculations, which can calculate only a very small region, albeit with a high degree of accuracy. However, we did use the Amber program (version 9.0, University of California, San Francisco) ${ }^{51,52}$ implemented with a semiempirical PM3 method, or Gromacs (version 3.3) ${ }^{53-55}$, implemented with the PM3 method, which we employed in addition to the standard program settings. To determine in detail the means by which the ATP hydrolysis reaction progresses, we use MD calculations of both the original prehydrolysis structures and a nearly identical prehydrolysis structure, modified so that only the position of one of the protons of the lytic water is different: the proton is moved from the lytic water to candidates of the proton destination.

\section{Our Future Studies}

Our ongoing studies will employ QM/MM-MD calculations of other structures of the transition state and will ultimately perform computer simulations of the whole process of ATP hydrolysis, including the intake of ATP into the ATP catalytic pocket, the positioning of ATP within the pocket, the hydrolysis of ATP, and the discharge of products from the pocket. In addition, we hope to pursue the study of structural changes of myosin corresponding to the hydrolysis. This approach will be facilitated by experimental studies of the point mutation of myosin by Onishi, et al., ${ }^{48-50}$ and Sutoh, et $\mathrm{al}^{56}$ We also hope to elucidate the molecular mechanism of muscle contraction via studies of the interaction with actin and, more broadly, to elucidate the molecular mechanism of other molecular motors, such as kinesin and $\mathrm{F}_{1}$-ATPase, and those involved in the highly efficient energy transduction of biological systems.

\section{References}

1. Goldman YE: Wag the tail: Structural dynamics of actomyosin. Cell 1998; 93: 1-4.

2. Block SM: Kinesin: What gives? Cell 1998; 93: 5-8.

3. Lohman TM, Thorn K, Vale RD: Staying on track: Common features of DNA helicases and microtubule motors. Cell 1998; 93: 9-12.

4. Gelles J, Landick R: RNA polymerase as a molecular motor. Cell 1998; 93: 13-16.

5. DeRosier DJ: The turn of the screw: The bacterial flagellar motor. Cell 1998; 93: 17-20.

6. Kinosita K, Yasuda R, Noji H, Ishiwata S, Yoshida M: F-ATPase: A rotary motor made of a single molecule. Cell 1998; 93: 21-24.

7. Alberts B, Bray D, Lewis J, Raff M, Roberts K, Watson JD: Molecular Biology of the Cell 3rd ed, 1994; Garland Pub., New York.

8. Tonomura Y: Muscle Proteins, Muscle Contraction and Cation Transport, 1972; University of Tokyo Press, Tokyo.

9. Holmes KC: Muscle proteins: Their actions and interactions. Curr Opin Struct Biol 1996; 6: 781-789.

10. Cooke R: The actomyosin interaction in striated muscle. Physiol Reviews 1997; 77: 671-697.

11. Metropolis N, Ulam S: The Monte Carlo method. J Am Stat Assoc 1949; 44: 335-341.

12. Alder BJ, Wainwright TE: Studies in molecular dynamics. II. Behavior of a small number of elastic spheres. J Chem Phys 1960; 33: 1439-1451.

13. Alder BJ, Wainwright TE: Phase transition in elastic disks. Phys Rev 1962; 127: 359-361.

14. Fukui K, Morokuma K, Nagata C: A molecular orbital treatment of phosphate bonds of biochemical interest. I. Simple LCAO MO treatment. Bull Chem Soc Japan 1960; 33: 1214-1219.

15. Fukui K, Imamura A, Nagata C: A molecular orbital treatment of phosphate bonds of biochemical interest. II. Metal chelates of adenosine triphosphate. Bull Chem Soc Japan 1963; 36: 1450-1453.

16. Kothekar V, Tyagi RS, Mishra RK: Molecular orbital calculations of magnesium complexes with ATP \& ADP. Indian J Biochem Biophys 1973; 10: 279-282.

17. Kothekar V, Mishra RK: Molecular orbital calculations on ATP \& ADP: Part II-A comparison of the role of external cations $\left(\mathrm{Mg}^{2+}, \mathrm{Ca}^{2+}, \mathrm{Na}^{+}\right)$. Indian J Biochem Biophys 1974; 11: 259-262.

18. Yoshikawa K, Shinohara Y, Terada H, Kato S: Why is $\mathrm{Mg}^{2+}$ necessary for specific cleavage of the terminal phosphoryl group of ATP? Biophys Chem 1987; 27: 251-254. 
19. Tajima M, Honda M: Molecular orbital study on the hydrolysis of adenosine triphosphate: The role of a cation as catalyst. J Mol Struct (Theochem) 1991; 228: 201-208.

20. Fukui K, Yonezawa T, Nagata C, Shingu H: Molecular orbital theory of orientation in aromatic heteroatomic, and other conjugated molecules. J Chem Phys 1954; 22: 1433-1442.

21. Pople JA, Beveridge DL: Approximate Molecular Orbital Theory, 1970; McGraw-Hill, New York.

22. http://qcpe.chem.indiana.edu/

23. Kagawa H, Nagai Y, Mori K: Optimized structures of ionized adenosine triphosphate (in Japanese). Bull Lib Arts \& Sci Nippon Med Sch 1997; 22: 79-93.

24. Kagawa H, Mori K: Molecular orbital study of the interaction between $\mathrm{MgATP}$ and the myosin motor domain: The highest occupied molecular orbitals indicate the reaction site of ATP hydrolysis. J Phys Chem B 1999; 103: 7346-7352.

25. Fukui K: Theory of Orientation and Stereoselection, 1970; Springer, Berlin.

26. Fukui K, Fujimoto H: Frontier Orbitals and Reaction Paths: Selected Papers of Kenichi Fukui (Fukui K, Fujimoto H, eds), 1997; World Scientific, Singapore.

27. Stewart JJP: Optimization of parameters for semiempirical methods. I. Method. J Comput Chem 1989; 10: 209-220.

28. Stewart JJP: Optimization of parameters for semiempirical methods. II. Applications. J Comput Chem 1989; 10: 221-264.

29. Stewart JJP: Optimization of parameters for semiempirical methods. III. Extension of PM3 to Be, $\mathrm{Mg}, \mathrm{Zn}, \mathrm{Ga}, \mathrm{Ge}, \mathrm{As}$, Se, Cd, In, Sn, Sb Te, Hg, Tl, Pb, and Bi. J Comput Chem 1991; 12: 320-341.

30. http://software.fujitsu.com/jp/winmopac/

31. Fisher AJ, Smith CA, Thoden JB, et al.: X-ray structures of the myosin motor domain of Dictyostelium discoideum complexed with MgADP$\mathrm{BeF}_{x}$ and $\mathrm{MgADP}-\mathrm{AlF}_{4}^{-}$. Biochemistry 1995; 34: 8960-8972.

32. Gulick AM, Bauer CB, Thoden JB, Rayment I: X-ray structures of the MgADP, MgATP $\gamma$, and MgAMPPNP complexes of the Dictyostelium discoideum myosin motor domain. Biochemistry 1997; 36: 11619-11628.

33. Smith CA, Rayment I: X-ray structure of the magnesium(II)-ADP-vanadate complex of the Dictyostelium discoideum myosin motor domain to 1.9 A resolution. Biochemistry 1996; 35: 5404-5417.

34. Okimoto N, Yamanaka K, Ueno J, Hata M, Hoshino T, Tsuda M: Theoretical studies of the ATP hydrolysis mechanism of myosin. Biophys J 2001; 81: 2786-2794.

35. Minehardt TJ, Marzari N, Cooke R, Pate E, Kollman PA, Car R: A classical and ab initio study of the interaction of the myosin triphosphate binding domain with ATP. Biophys J 2002; 82: 660-675.

36. Schwarzl SM, Smith JC, Fischer S: Insights into the chemomechanical coupling of the myosin motor from simulation of its ATP hydrolysis mechanism. Biochemistry 2006; 45: 5830-5847.

37. Frisch MJ, Trucks GW, Schlegel HB, et al.: Gaussian 98, Revision A.7, 1998; Gaussian, Pittsburgh.

38. http://www.gaussian.com/
39. Pearlman DA, Case DA, Caldwell JW, et al.: AMBER, a package of computer programs for applying molecular mechanics, normal mode analysis, molecular dynamics and free energy calculations to simulate the structural and energetic properties of molecules. Comput Phys Commun 1995; 91: 1-41.

40. Car R, Parrinello M: Unified approach for moleculardynamics and density-functional theory. Phys Rev Lett 1985; 55: 2471-2474.

41. Case DA, Pearlman DA, Caldwell JW, et al.: AMBER 6, 1999; University of California, San Francisco.

42. Dominguez R, Freyzon Y, Trybus KM, Cohen C: Crystal structure of a vertebrate smooth muscle myosin motor domain and its complex with the essential light chain: Visualization of the pre-power stroke state. Cell 1998; 94: 559-571.

43. Schmidt MW, Baldridge KK, Boatz JA, et al:: General atomic and molecular electronic structure system. J Comput Chem 1993; 14: 1347-1363.

44. http://www.msg.ameslab.gov/GAMESS/

45. Brooks BR, Bruccoleri RE, Olafson BD, States DJ, Swaminathan S, Karplus M: CHARMM: A program for macromolecular energy, minimization, and dynamics calculations. J Comput Chem 1983; 4: 187217.

46. http://www.charmm.org/

47. Onishi H, Mochizuki N, Morales MF: On the myosin catalysis of ATP hydrolysis. Biochemistry 2004; 43: 3757-3763.

48. Onishi H, Morales MF, Kojima S, Katoh K, Fujiwara $\mathrm{K}$ : Functional transitions in myosin: Role of highly conserved Gly and Glu residues in the active site. Biochemistry 1997; 36: 3767-3772.

49. Onishi H, Kojima S, Katoh K, Fujiwara K, Martinez HM, Morales MF: Functional transitions in myosin: Formation of a critical salt-bridge and transmission of effect to the sensitive tryptophan. Proc Natl Acad Sci USA 1998; 95: 6653-6658.

50. Onishi H, Ohki T, Mochizuki N, Morales MF: Early stages of energy transduction by myosin: Roles of Arg in switch I, of Glu in switch II, and of the saltbridge between them. Proc Natl Acad Sci USA 2002; 99: 15339-15344.

51. Case DA, Darden TA, Cheatham TE III, et al: AMBER 9, 2006; University of California. San Francisco.

52. http://amber.scripps.edu/

53. Lindahl E, Hess B, Van der Spoel D: GROMACS 3.0: A package for molecular simulation and trajectory analysis. J Mol Mod 2001; 7: 306-317.

54. Van der Spoel D, Lindahl E, Hess B, Groenhof G, Mark AE, Berendsen HJC: GROMACS: Fast, flexible and free. J Comput Chem 2005; 26: 1701-1718.

55. http://www.gromacs.org/

56. Sasaki N, Sutoh K, Sasaki N: Structure-mutation analysis of the ATPase site of Dictyostelium discoideum myosin II. Adv Biophys (Japan) 1998; 35: $1-24$.

(Received, December 13, 2006)

(Accepted, December 29, 2006) 